\title{
Intervenção fonoaudiológica precoce em afasia infantil decorrente de um acidente vascular cerebral: relato de caso
}

\author{
Early speech-language intervention in \\ childhood aphasia after a stroke: case report
}

\section{Intervención fonoaudiológica precoz en afasia infantil debido a un accidente vascular cerebral: reporte de un caso}

\author{
Natalia Caroline Favoretto* \\ Natalia Gutierrez Carleto* \\ Paula Grandini Cunha* \\ Vanessa Clivelaro Bertassi Panes* \\ Adriano Yacubian Fernandes* \\ Dionísia Aparecida Cusin Lamônica* \\ Magali de Lourdes Caldana*
}

\section{Resumo}

O Acidente Vascular Cerebral é menos frequente em crianças do que em adultos, porém possui um poder devastador para o futuro destes indivíduos, mesmo com indícios de melhor recuperação das alterações. O objetivo deste estudo é descrever os achados fonoaudiológicos de um caso de Acidente Vascular Cerebral infantil, evidenciando a evolução após 12 meses de terapia fonoaudiológica, iniciada

\footnotetext{
* Faculdade de Odontologia de Bauru, Universidade de São Paulo, Bauru, SP, Brasil Contribuição dos autores:

NCF: avaliação do sujeito e elaboração do planejamento terapêutico fonoaudiológico do caso clínico, e elaboração do artigo; NGC: avaliação do sujeito e elaboração do planejamento terapêutico fonoaudiológico do caso clínico, e elaboração do artigo; PGC: avaliação do sujeito e elaboração do planejamento terapêutico fonoaudiológico do caso clínico, e elaboração do artigo; VCBP: discussões interdisciplinares;

AYF: análise de neuroimagens; elaboração do artigo;

DACL: co-orientação do estudo;

MLC: supervisão dos atendimentos fonoaudiológicos; elaboração do artigo.

E-mail para correspondência: Natalia Caroline Favoretto - natalia.favoretto@yahoo.com Recebido: 07/03/2017

Aprovado: 29/08/2017
} 
precocemente, após lesão neurológica adquirida. Trata-se de um indivíduo de 11 anos, gênero masculino, com normalidade de desenvolvimento neuropsicomotor e independência para as atividades funcionais durante a primeira infância. Em março de 2015 sentiu um mal súbito, foi encaminhado para o hospital de urgência e diagnosticado com Acidente Vascular Cerebral, do tipo hemorrágico, em região frontoparietotemporal esquerda. Foram realizados procedimentos cirúrgicos, e a internação durou 25 dias. No momento da alta hospitalar houve a orientação sobre a necessidade de atendimento fonoaudiológico. A primeira avaliação de linguagem evidenciou afasia adquirida do tipo emissiva, conforme as classificações propostas para crianças. Foram realizadas 91 sessões de terapia fonoaudiológica, com duração de 50 minutos, pelo período de 12 meses. Ao analisar as habilidades formais de linguagem que permaneceram alteradas, observa-se que as relacionadas à leitura e escrita são as de maior significância, interferindo no desempenho escolar e desenvolvimento comunicativo. Assim, além do atendimento fonoaudiológico até que seja possível uma comunicação funcional, é importante que haja o apoio escolar.

Palavras-chave: Fonoaudiologia; Linguagem; Acidente Vascular Cerebral; Crianças.

\section{Abstract}

Stroke is less common in children than in adults, but it has a devastating power for the future of these individuals, even with signs of better recovery from the changes. The aim of this study is to describe the speech therapy findings of a case of stroke child, showing the progress after 12 months of speech therapy initiated early after acquired neurological injury. This is an individual of 11 years old, male, with neurodevelopment normality and independence to the functional activities during early childhood. In March of 2015, he suffered a sudden illness, was referred to the emergency hospital and diagnosed with hemorrhagic stroke, in the left frontoparetotemporal region. Surgical procedures were performed, and hospitalization lasted 25 days. At the time of hospital discharge, there was guidance about the need for speech therapy care. In the first evaluation of language, there was evidence of acquired aphasia of the emissive type, according to the classifications proposed for children. A total of 91 speech therapy sessions lasting 50 minutes were performed for 12 months. When analyzing formal language skills that have remained altered, it is observed that those related to reading and writing are those of greater significance, interfering in school performance and communicative development. Thus, in addition to speech and language therapy until functional communication is possible, it is important that there be school support.

Keywords: Speech, Language and Hearing Sciences; Language; Stroke; Child.

\section{Resumen}

El accidente cerebrovascular es menos común en niños que en adultos, pero tiene un poder devastador para el futuro de estes individuos aun que se tenga indicios de mejor recuperación de las alteraciones. El objetivo de este estudio es describir los hallazgos fonoauiológicos de un caso de Accidente Vascular Cerebral Infantil, que muestra el progreso después de 12 meses de terapia fonoaudiológica iniciada precozmente, en un caso de lesión neurológica adquirida. Se trata de un individuo de 11 años, de sexo masculino, con normalidad el desarrollo neuropsicomotor y independencia de las actividades funcionales durante la primera infancia. En marzo el año 2015 sintió un mal súbito, fue remitido al hospital de emergencia y diagnosticado como Accidente Vascular Cerebral, de tipo hemorrágico en la región frontoparietotemporal izquierda. Se realizaron procedimientos quirúrgicos y la hospitalización duró 25 días. En el momento del alta hospitalaria hubo orientación sobre la necesidad de tratamiento fonoaudiológico. La primera evaluación del lenguaje mostró afasia adquirida de tipo emisivo, de acuerdo con las clasificaciones propuestas para los niños. Se realizaron 91 sesiones de terapia fonoaudiológica, con una duración de 50 minutos, el período de 12 meses. Mediante el análisis de las habilidades lingüísticas formales que permanecieron cambiada, se observa que las relacionadas con la lectura y la escritura son las más significativas, porque interfieren con el desempeño escolar y desarrollo comunicativo. Por lo tanto, además de la terapia fonoaudiologoca hasta que una comunicación funcional sea posible, es importante el apoyo escolar.

Palabras clave: Fonoaudiologia; Lenguaje; Acidente Vascular Cerebral; Niño. 


\section{Introdução}

O Acidente Vascular Cerebral (AVC) pode ser definido como o rápido desenvolvimento de sinais clínicos decorrentes de distúrbios focais ou globais da função cerebral, com sintomas com duração superior a 24 horas, de origem vascular, resultando alterações cognitivas e sensório-motoras, de acordo com a área e a extensão da lesão ${ }^{1}$.

Estima-se que, uma em cada cinco mulheres e, um em cada quatro homens poderão sofrer um AVC até os 85 anos de idade, e este risco aumenta após os 55 anos $^{2}$. Quando se trata de crianças, a variável é de dois a oito casos a cada 100.000 crianças até a idade de 14 anos, sendo que são acontecimentos raros e isolados, de caráter importante pela gravidade de suas complicações e variedade de etiologias. Algumas das principais causas de AVC em crianças são: cardiopatia congênita, doença hematológica (anemia falciforme, policitemia, púrpura alo-imune do recém-nascido), doença auto-imune, coagulopatias, vasculite, anomalias vasculares, infartos venosos, doenças metabólicas, vasoespasmo, traumas, desidratação, neoplasias, entre outras ${ }^{3,2}$. $\mathrm{O} A V C$ é menos frequente em crianças do que em adultos, porém quando ocorre, possui um poder devastador para o futuro destes indivíduos, mesmo com indícios de melhor recuperação das alterações por esta população ${ }^{4}$.

O AVC pode ser dividido em dois tipos: isquêmico e hemorrágico. O AVC isquêmico (AVCI) é caracterizado pela obstrução de um vaso sanguíneo que bloqueia o fluxo de sangue para as células cerebrais, não ocorrendo o recebimento necessário dos nutrientes indispensáveis ao metabolismo de suas

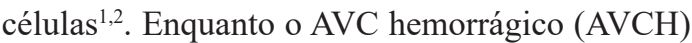
consiste no rompimento de um vaso sanguíneo, gerando o extravasamento de sangue e aumento da pressão intracraniana ${ }^{1}$. O tipo de $\mathrm{AVC}$ mais comum, em qualquer faixa etária, é o $\mathrm{AVCI}^{5}$.

Após o acometimento, independente do tipo de AVC, um dos danos mais comuns ao sujeito é o distúrbio adquirido de linguagem, denominado afasia, que é caracterizada por alterações linguísticas de origem discursiva e articulatória, produzida por lesão focal no sistema nervoso central, em áreas responsáveis pela linguagem, podendo ou não estar associada a outras alterações cognitivas, tornando o indivíduo dependente de recursos auxiliares para a produção e/ou interpretação da linguagem ${ }^{6}$.
Para as crianças, a afasia pode ser subdivida em desenvolvimental e adquirida. Se ocorrer antes dos dois anos de idade a afasia é considerada desenvolvimental, se ocorrer após, adquirida. A idade de dois anos é um marcador de tempo devido ao fato de que, nesta faixa etária, a criança deve ter desenvolvido aspectos mínimos da linguagem, como alguns fonemas e a capacidade de produzir sentenças simples ${ }^{7}$. A classificação dos tipos de afasia na infância também pode se diferenciar de acordo com as manifestações linguísticas, de forma mais abrangente, dividindo-se em afasia emissiva e receptiva, considerando os parâmetros de expressão e compreensão, respectivamente ${ }^{8}$.

O tipo e a gravidade da afasia estão diretamente relacionados ao local e extensão da lesão neurológica adquirida, sendo a relação entre áreas cerebrais e a linguagem alvo de estudos há anos, mostrando principalmente a importância do hemisfério esquerdo, mais especificamente as áreas de Broca e Wernicke 9 . Outro fato conhecido é que regiões extras influenciam diretamente e indiretamente nas habilidades linguísticas, bem como o hemisfério direito, responsável pelos aspectos paralinguísticos da comunicação ${ }^{10}$.

Considerando os aspectos citados, ressalta-se que, em todos os casos de AVC, é de suma importância considerar a etiologia, história clínica, local e extensão da lesão, relacionando tais fatores com as alterações apresentadas pelos sujeitos acometidos, principalmente naqueles em que houver a presença da afasia, pois cada caso é único e possui diferentes manifestações que podem atingir todos os níveis formais da linguagem, dentre eles a fonologia, morfologia, sintaxe, semântica, pragmática e o léxico.

O objetivo do presente estudo é descrever os achados fonoaudiológicos de um caso de AVC infantil, evidenciando a evolução após 12 meses de terapia fonoaudiológica, iniciada precocemente, após lesão neurológica adquirida e, dessa forma, reforçar a importância da intervenção precoce, a fim de proporcionar um prognóstico favorável à pessoa com afasia.

\section{Apresentação do caso clínico}

O trabalho fundamentou-se em um estudo de caso clínico de um paciente atendido na Clínica de Fonoaudiologia da instituição de origem. O estudo não foi enviado ao Comitê de Ética em Pesquisa com Seres Humanos (CEP), pois ao iniciar o trata- 
mento fonoaudiológico, o responsável pelo paciente assinou o Termo de Autorização para Utilização dos Dados, como previsto e concedido pela própria instituição, dispensando a aprovação pelo CEP.

O paciente compareceu à Clínica de Fonoaudiologia acompanhado dos pais, que participaram da anamnese e auxiliaram no relato da seguinte história clínica: indivíduo de 11 anos de idade, gênero masculino, com normalidade de desenvolvimento neuropsicomotor e independência para as atividades funcionais durante a primeira infância. Em março de 2015 sentiu mal estar seguido de síncope, foi socorrido em um breve período de tempo, aproximadamente 10 minutos, e encaminhado para o hospital, onde foi realizada avaliação neurológica por meio de tomografia computadorizada de crânio, com cortes axiais, utilizando o aparelho Multi Slice, que fornece reconstruções multiplanares, sem a infusão intravenosa do meio de contraste iodado. Imediatamente, foi diagnosticado $\mathrm{AVCH}$ em região fronto-parietotemporal esquerda e realizada neurocirurgia para conter a hemorragia, sendo que, após quatro dias, houve novo foco hemorrágico acom- panhado de edema cerebral, com a necessidade de outro procedimento cirúrgico para descompressão intra-craniana. A alta hospitalar aconteceu 25 dias após o acometimento, com orientações sobre a necessidade de atendimento fonoaudiológico.

A primeira avaliação de linguagem ocorreu em abril de 2015, por meio do Teste de Reabilitação das Afasias - versão adaptada ${ }^{11}$ e do Token Test - versão adaptada para crianças ${ }^{12}$, evidenciando a classificação de afasia adquirida do tipo emissiva, conforme as classificações propostas para crianças ${ }^{7,8}$.

Foram realizadas 91 sessões de terapia fonoaudiológica, com duração de 50 minutos, pelo período de 12 meses. A intervenção foi realizada por três terapeutas distintas, com revezamento de terapeutas, respeitando o ciclo da grade curricular do curso de graduação em Fonoaudiologia, sendo todas as sessões supervisionadas por uma única docente especialista na área. Os planejamentos terapêuticos foram abrangentes para atender todas as alterações fonoaudiológicas encontradas na avaliação (tabela 1).

Tabela 1. Avaliações pré e pós-intervenção fonoaudiológica

\begin{tabular}{ccc}
\hline Habilidades & $\begin{array}{c}\text { Resultados } \\
\text { pré-intervenção fonoaudiológica }\end{array}$ & $\begin{array}{c}\text { Resultados } \\
\text { pós-intervenção fonoaudiológica }\end{array}$ \\
\hline Fluência & Inalterado & Inalterado \\
Leitura & Alterado & Alterado \\
Escrita & Alterado & Alterado \\
Nomeação & Alterado & Inalterado \\
Repetição & Alterado & Inalterado \\
Evocação & Alterado & Inalterado \\
Compreensão da LO & Inalterado & Inalterado \\
Compreensão da LE & Alterado & Alterado \\
\hline
\end{tabular}

Legenda: LO: linguagem oral. LE: linguagem escrita

\section{Discussão}

Dentre os tipos de AVC, o isquêmico é o mais comum independente da faixa etária, relacionando-se com fatores de risco de ordens hereditárias. Em contrapartida, o AVCH está mais relacionado a problemas de malformação vascular, estando presentes ou ausentes outros fatores de risco adquiridos ou hereditários ${ }^{13}$.

Algumas das anomalias vasculares são as malformações arteriais, arteriovenosas e fístulas arteriovenosas. Dentre estas, merece destaque a malformação arteriovenosa, caracterizada por fístulas arteriovenosas que possuem um epicentro denominado ninho, composto por artérias que nutrem e aumentam o volume das veias, podendo estar presentes no nascimento ou se evidenciarem durante o desenvolvimento do indivíduo, sendo que elas não diminuem ou regridem de forma espontânea. As mudanças hormonais para a puberdade ou traumas, podem mobilizar o crescimento da malformação arteriovenosa, pois as veias de drenagem se tornam mais evidenciadas, tortuosas e distendidas, podendo ocasionar uma hemorragia ${ }^{14}$. Alguns 


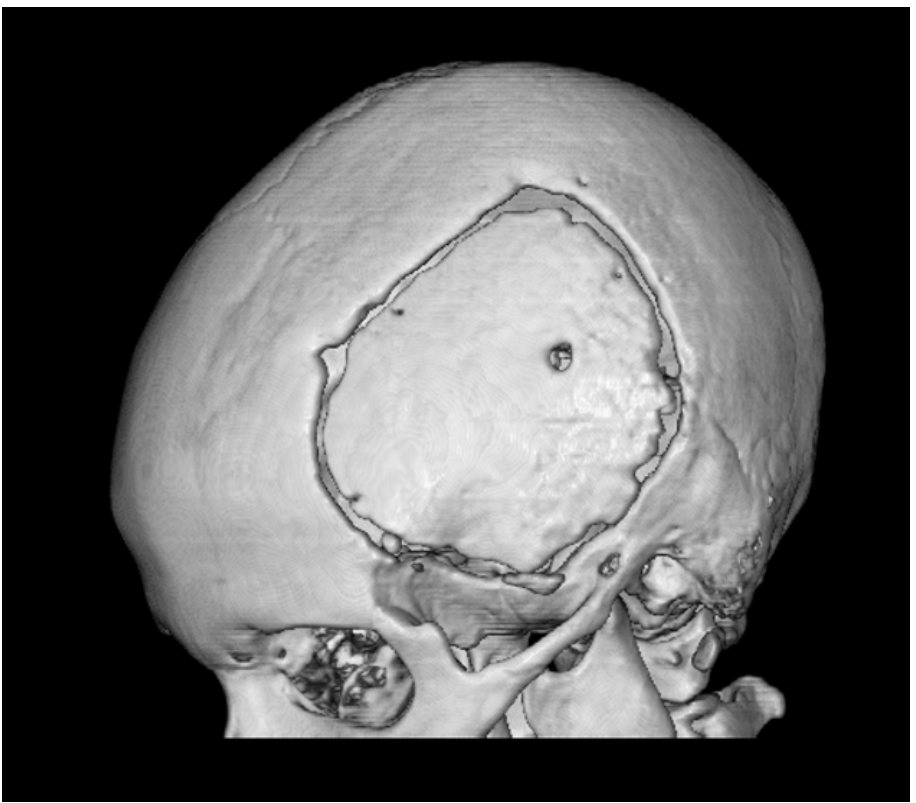

Figura 1. Controle pós-craniectomia na região fronto-parietotemporal esquerda

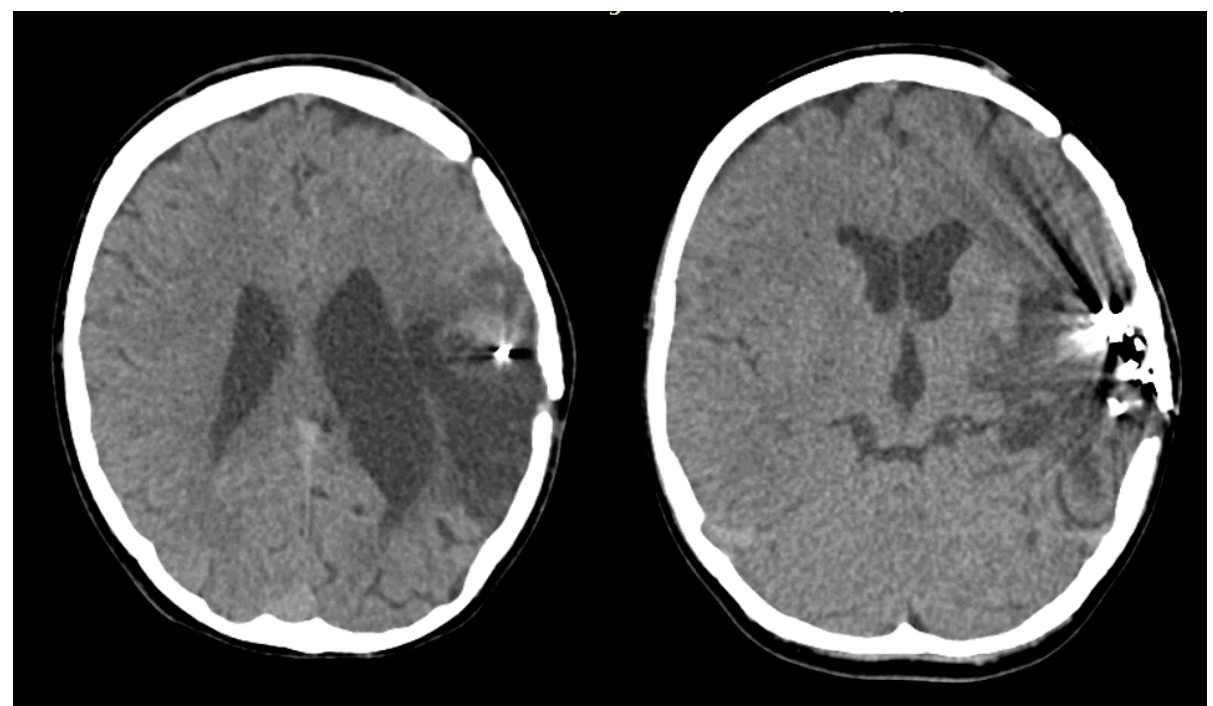

Figura 2. Tomografia Computadorizada de Crânio, após craniectomia, destacando área hipoatenuante de aspecto sequelar na região fronto-parietotemporal esquerda

critérios estabelecidos na década de 60 ainda são utilizados e consideram o início da puberdade aos oito anos para as meninas e em nove anos para os meninos ${ }^{15}$. Apesar de o tipo mais comum de AVC ser o isquêmico, o caso clínico abordado relata um AVC do tipo hemorrágico ocorrido durante o período de puberdade e resultante de malformação arteriovenosa, que é o principal fator de risco para este tipo de AVC na infância, o que corrobora com os achados da literatura.

A relação entre tipo, local e extensão da lesão e manifestações na comunicação do indivíduo merecem atenção, visto que, os lobos temporal, parietal e região pré-frontal do hemisfério esquerdo atuam de forma direta nos processos léxico-semânticos ${ }^{16}$, havendo forte correlação entre as 
áreas têmporo-parietais e as tarefas de discurso, repetição, nomeação, compreensão da escrita e da leitura ${ }^{9}$. A lesão cerebral do paciente compreende as áreas supracitadas, exceto região pré-frontal, o que reforça a concordância entre manifestações linguísticas e locais de lesões neurológicas adquiridas do caso estudado.

O efeito da lesão cerebral em crianças é diferente do efeito em adultos ${ }^{17}$. Caso uma criança seja acometida por uma lesão cerebral que comprometa completamente seu hemisfério dominante, poderá apresentar afasia, mas com maior chance de recuperação da linguagem, pois a reorganização cerebral, decorrente da neuroplasticidade, encontra-se em maior potencial comparado à individuos adultos e idosos, fato que possivelmente colaborou no processo de reabilitação neste relato de caso, visto que o paciente iniciou a intervenção fonoaudiológica aos 11 anos de idade.

Em relação à reabilitação precoce, quando o paciente é submetido a terapia desde a fase aguda do AVC, a recuperação pode ser até duas vezes maior quando comparada a recuperação espontânea. Além disso, se a terapia fonoaudiológica for iniciada após a fase aguda, os resultados podem ser menos expressivos, ainda que significativos ${ }^{18}$. Levando em consideração que o tratamento fonoaudiológico inciou-se precocemente, em torno de um mês após o acometimento cerebral, foi possível observar melhora significativa nas habilidades comunicativas, visto que, ao comparar as avaliações pré e pós-intervenção fonoaudiológica, nota-se que, de seis habilidades alteradas, restaram apenas três que não apresentaram melhora expressiva.

Ao analisar as habilidades formais de linguagem que permaneceram alteradas após 12 meses de intervenção, observa-se que as relacionadas à leitura e escrita são as de maior significância, interferindo no desempenho escolar e desenvolvimento comunicativo, sendo, portanto, necessário o acompanhamento fonoaudiológico unido ao apoio escolar. Dessa forma, por mais que a afasia na infância tenha um melhor prognóstico, essa criança precisará de acompanhamento por um período maior, compreendendo este por toda sua infância e até a aquisição total da linguagem em seu período escolar, devido às possíveis dificuldades ${ }^{8}$, o que reforça a importância e a necessidade de comunicação e parceria com toda a equipe escolar, promovendo qualidade no processo de reabilitação.

\section{Conclusão}

Um acometimento cerebral infantil não é algo comum e casual. Ao longo dos anos, a variável de crianças atingidas vem aumentando exponencialmente, por isso, é necessário que haja mais estudos, uma vez que sua etiologia, diagnóstico e prognóstico são desafiadores. É importante que este indivíduo seja assistido de forma precoce, reforçando a importância da intervenção em período de maior neuroplasticidade, e que seja acompanhado até atingir uma comunicação funcional. Além disso, destaca-se a importância da associação entre os achados fonoaudiológicos e neurológicos para que ocorra maior sucesso nos processos de reabilitação da linguagem.

\section{Referências}

1. World Health Organization (WHO). Neurological disorders: public health challenges [Acesso em 20 out de 2016]. Disponíel em: http://www.who.int/mental_health/neurology/neurological_ disorders_report_web.pdf.

2. Liégio LM, Borges DM, Faria PHC, Pacheco LP, Martins PP. Atendimento ao acidente vascular encefálico infantil: perspectiva de neuropediatras. Enfermaria Global 2011; 4(22); $1-14$.

3. Mekitarian Filho E, de Carvalho WB. Stroke in children. J Pediatr (Rio J). 2009; 85(6): 469-79.

4. Avila, LN. A linguagem em crianças e adolescentes acometidos por acidente vascular cerebral infantil. Porto Alegre. Dissertação [mestrado] - Universidade Federal Rio Grande do Sul. Faculdade de Medicina; 2009.

5. Rotta NT \& Ranzan J. Acidente vascular cerebral. In: Rotta NT, Ohlweiler L, Riesgo RS. Rotinas em Neuropediatria. Porto Alegre: Artmed; 2005.p.123-130.

6. Coudry MIH. Diário de Narciso: discurso e afasia: análise discursiva de interlocuções com afásicos. 2. ed. São Paulo: Martins Fontes; 1996.

7. Van Hout A. Acquired afasia in childhooh. In Segalowits SJ, Rapin I. Hanbook of Neuropsychology, vol 8. 2. ed, Amsterdam: Elsevier, 2003.

8. Ortiz, KZ. Distúrbios neurológicos adquiridos - linguagem e cognição. Barueri: Manole; 2005.p.60-61,

9. Vieira ACC, Roazzi A, Queiroga BM, Asfora R, Valença MM. Afasias e áreas cerebrais: argumentos prós e contras à perspectiva localizacionista. Psicol. Reflex e Crit. 2011; 24(3): 588-96.

10. Fonseca RP, Wagner GP, Rinaldi J, Parente MAMP. $\mathrm{O}$ envelhecimento influencia as habilidades pragmáticas, léxico-semânticas e prosódicas do hemisfério direito? Estudos interdisciplinares sobre o envelhecimento 2007; 12:53-79.

11. Jakubovicz R. Teste de Reabilitação das Afasias. Rio de Janeiro: Revinter, 2005. 
12. Di Simoni F. The Token Test for children. Boston: Teaching Resources Corporation, 1978.

13. Gandra JD, Bastos MAR. Fatores de risco para ocorrência de acidente vascular encefálico em indivíduos de 0 a 20 anos. Enferm Rev 2014 jan/abr; 17(1): p.61-78.

14. Gontijo B, Pereira LB, Silva CMR. Malformações vasculares. Na Bras Dermatol 2004 jan/fev;79(1):p.7-25.

15. Marshall WA, Tanner JM. Variations in the pattern of pubertal changes in girls. Arch Dis Child 1969; 44:p.291-303.
16. Pereira JR, Reis AM, Magalhães Z. Neuroanatomia funcional: Anatomia das áreas activáveis nos usuais paradigmas em ressonância magnética funcional. Acta Med Port 2003; 16:p.107-16.

17. Thompson RF. O cérebro - uma introdução à neurociência. 3.ed. Santos: Santos; 2005. p.444.

18. Robey RR. The efficacy of treatment for aphasic persons: a meta-analysis. Brain and Language. 1994; 47(4): p.582-608. 